\title{
Analyse de l'impact des normes IFRS sur l'estimation du risque de défaillance des groupes cotés : Une étude exploratoire sur le marché français
}

\author{
Lionel ESCAFFRE et Olivier RAMOND
}

\begin{abstract}
Résumé : Selon le principe de continuité d'exploitation, une entreprise est réputée être en continuité d'activité en l'absence de toute information permettant d'établir le contraire. Durant les trente dernières années, la littérature académique s'est efforcée d'étudier les informations aidant potentiellement au travail d'appréciation du risque de défaut d'une entreprise. Ces travaux se sont souvent révélés prolifiques en utilisant initialement des modèles comptables et plus récemment une approche optionnelle. Néanmoins, la transition des normes françaises vers le référentiel IFRS au $1^{\text {er }}$ janvier 2005 peut avoir impacté cette méthodologie. A l'aide d'un échantillon d'entreprises industrielles françaises constitutives de l'indice SBF 120, cette étude examine, à partir d'une analyse exploratoire fondée sur les sensibilités, l'impact de la transition au référentiel IFRS sur l'estimation du risque de défaut tel qu'approximé par les principaux modèles comptables (Altman (1968); Ohlson (1980) et Zmijewski (1984)) et optionnels (Merton (1974) et Vassalou et Xing (2004)). Les résultats empiriques indiquent que ceteris paribus l'application des normes IFRS par les sociétés cotées échantillonnées a, en moyenne, diminué, de manière significative, leur risque de défaut. Nous en concluons qu'à l'opposé des normes françaises reposant sur une approche redditionnelle de la comptabilité, le passage au référentiel IFRS fondé sur une approche prospective implique une nécessaire révision des hypothèses sous-jacentes aux modèles prédictifs du risque de défaut.
\end{abstract}

Mots-clés : Continuité d'exploitation, cadre conceptuel de l'IASB, modèles de risque de défaut, information comptable, transition aux normes IAS / IFRS.

\section{Study of the impact of IFRS standards on the listed firms' default risk measurement: An exploratory study on the French capital market}

Abstract: Under the going concern principle, an entity is considered as a going concern in the absence of information proving the contrary. Over the last thirty years, researchers have extensively examined the information relevant to default risk assessment. These empirical works have turned to be highly conclusive while first adopting accounting-based and more recently option-pricing based approaches. However, the 2005 transition from French GAAPs towards IFRS framework may have impacted the valuation methodologies documented previously. Using a sample made of SBF 120 industrial companies, this study examines the impact of the IFRS transition on the default risk assessment as gauged by the main accounting-based (i.e. Altman (1968); Ohlson (1980) and Zmijewski (1984)) and optionbased models (i.e. Merton (1974) and Vassalou et Xing (2004)). Our empirical results suggest that ceteris paribus sampled listed firms exhibit on average a significant smaller default risk under IAS than under French GAAPs. This finding underlines the fact that contrary to reddition-oriented French GAAPs, the transition to the prospective-based IFRS standards necessarily implies a reconsideration of the hypotheses underlying the common default risk models.

Keywords: Going concern, IASB conceptual framework, default risk models, accounting information, IAS / IFRS transition. 


\section{Analyse de l'impact des normes IFRS sur l'estimation du risque de défaillance des groupes cotés : Une étude exploratoire sur le marché financier français}

\section{Introduction}

En comptabilité, le risque de défaillance conduit les groupes à s'interroger sur la notion de continuité d'exploitation. Ce principe est énoncé dans la plupart des cadres comptables. En France, la continuité d'exploitation revêt une importance particulière car le commissaire aux comptes a l'obligation de déclencher une procédure $\mathrm{d}^{\prime}$ alerte ${ }^{1}$ dès lors qu'il «relève, à l'occasion de l'exercice de sa mission, des faits de nature à compromettre la continuité d'exploitation $^{2} »$. En tant que postulat, le principe de continuité d'exploitation vient résoudre, en apparence, le paradoxe qui consiste à communiquer une information comptable périodique relative à une activité continue. Il ne s'agit pas de prouver l'existence de la continuité mais d'évaluer les présomptions qui impliqueraient que cette convention doit être acceptée tout en s'assurant qu'il n'existe pas d'éléments probants justifiant sa remise en cause. Ainsi, de simples difficultés ou une appréciation à long terme de difficultés éventuelles ne sont pas constitutives d'une rupture de continuité d'exploitation que nous appellerons risque de défaillance.

En normes comptables internationales IAS / IFRS, l'établissement d'un diagnostic prévisionnel est nécessaire puisque «les états financiers sont normalement préparés selon l'hypothèse qu'une entité est en situation de continuité d'exploitation et poursuivra ses activités dans un avenir prévisible ${ }^{3} »$.

Ainsi les lecteurs des états financiers, tels que les analystes financiers, sont susceptibles d'appliquer des méthodologies d'analyse financière pour estimer le risque de défaillance. Pour ce faire, les utilisateurs doivent considérer toute une série de facteurs relatifs à la rentabilité actuelle et attendue, aux calendriers de remboursement des dettes et aux sources 
potentielles de remplacement de son financement ${ }^{4}$. La rentabilité des actifs ${ }^{5}$ et la structure de l'endettement, tel que l'endettement financier net, sont des agrégats majeurs en IFRS pour apprécier ce risque de défaillance.

De ce fait, en 2005, le passage aux normes IFRS des groupes cotés présentant des comptes consolidés modifie considérablement l'analyse du bilan et en conséquence, l'estimation du risque de défaillance. En effet, le recours à des justes valeurs fondées sur des valeurs de marché ou des valeurs actualisées est susceptible de générer des variations significatives sur les capitaux propres pour les actifs d'investissement et sur le compte de résultat pour les actifs de placement. Ces évolutions sont susceptibles de remettre en cause l'appréciation du risque de défaillance. Par ailleurs, le cadre comptable international repose faiblement sur la continuité d'exploitation puisqu'un bilan intégrant des justes valeurs suppose qu'il est possible de vendre à tout moment des actifs de l'entreprise séparément. Les recours aux Business Units en US GAAPs et aux Unités Génératrice de Trésorerie en normes IFRS illustrent cette conception de la valeur en comptabilité.

Suivant ces constats, la première adoption des normes internationales pour les exercices ouverts au $1^{\text {er }}$ janvier 2005 est l'occasion de s'interroger sur l'estimation du risque de défaillance à travers une analyse comparative relative à l'application des deux référentiels.

L'estimation du risque de faillite d'une entreprise industrielle ou commerciale s'effectue, traditionnellement, à l'aide d'indicateurs fondés sur des données comptables et financières issus eux-mêmes des états financiers. Cette méthode dite «traditionnelle » est souvent qualifiée d'incomplète par les auteurs ( $c f$. Barth et al., 1998) et appelle confirmation par l'utilisation d'une méthode basée sur les valorisations d'options (Merton, 1974). 
Ainsi, à l'aide d'un échantillon de sociétés cotées françaises appartenant à l'indice boursier SBF 120 ayant publié un double jeu de comptes IAS / French GAAPs à l'ouverture de l'exercice $2005^{6}$, nous nous proposons de tester si les modèles classiques de risque de défaillance (i.e. Altman, 1968 ; Ohlson, 1980 ; Zmijewski, 1984 ; Merton, 1974 ; Vassalou \& Xing (2004)) apportent les mêmes résultats selon le référentiel comptable considéré. Les résultats trouvés nous permettront de discuter de la pertinence des normes IAS / IFRS en matière de continuité d'exploitation au regard du référentiel comptable français.

La suite de l'étude procédera comme suit: la section 2 discute le concept de continuité d'exploitation au regard des dispositifs normatifs comptables anglosaxons (US GAAPs et IAS / IFRS) et national et développe notre question de recherche. La section 3 présente un descriptif et une analyse des principaux modèles d'estimation du risque de défaut, à partir desquels nous choisissons d'examiner la question de recherche. La section 4 décrit notre échantillon et présente une analyse des résultats empiriques de notre étude. Enfin, la section 5 fournit un résumé des principaux apports de l'étude et décline quelques remarques potentiellement utiles à la poursuite du travail d'investigation. 


\section{Perception du principe de continuité d'exploitation par les référentiels français et anglo-saxons (US GAAPs et IAS / IFRS)}

Depuis les travaux précurseurs de Beaver (1966), l'analyse des facteurs qui permettent d'estimer le risque de défaillance d'une entreprise a fait l'objet d'un grand nombre de recherches anglo-américaines en s'appuyant sur une démarche prédictive et positive. Le risque de défaillance est un risque dont l'apparition remet en cause la continuité d'exploitation de l'entité. Les référentiels comptables nationaux et internationaux ont, pour la plupart, inscrit dans leur cadre conceptuel le traitement comptable de l'information financière des entreprises en difficulté et proposent une définition du principe de continuité d'exploitation. Avant de discuter les résultats présentés par les études antérieures portant sur le lien entre information financière et risque de défaillance, une première analyse est donc consacrée aux dispositions normatives de l'information financière au regard du principe de continuité d'exploitation.

\subsection{Contexte normatif}

Depuis le $1^{\mathrm{er}}$ janvier 2005, les groupes français faisant appel public à l'épargne sur un marché réglementé sont dans l'obligation de publier leurs comptes consolidés en normes comptables internationales (IFRS). Les normes IFRS ont pour objectif d'harmoniser les cadres comptables nationaux, et à ce titre, il est particulièrement intéressant d'identifier le traitement de la prédiction des défaillances d'entreprises, d'une part, au sein de cadres nationaux tels que les normes françaises ou les normes américaines, et d'autre part, au sein du cadre international. 
2.1.1. Le cadre comptable français (PCG): une vision juridique de la continuité d'exploitation

Le problème de la défaillance est abordé dans le cadre comptable français sur un axe juridique par le code de commerce (Art L 123-20) : "Pour l'établissement des comptes annuels, le commerçant, personne physique ou morale, est présumé poursuivre ses activités. » Le Plan Comptable Général (art 120-1) confère une dimension comparative et prospective à la comptabilité en précisant lors de l'énonciation du principe de permanence des méthodes que «la comptabilité permet d'effectuer des comparaisons périodiques et d'apprécier l'évolution de l'entreprise dans une perspective de continuité d'activité ».

En application de ces principes, la Compagnie Nationale des Commissaire aux Comptes ${ }^{7}$ précise que «la continuité d'exploitation est une convention comptable de base selon laquelle, lors de l'arrêté des comptes annuels, il convient d'apprécier si l'entreprise est en activité, s'il n'existe aucun fait qui pourrait l'empêcher de rester en activité dans un avenir prévisible. Lorsque la continuité d'exploitation parait assurée, les comptes annuels sont arrêtés normalement, c'est-à-dire dans le respect des autres conventions comptables généralement admises. Lorsque la continuité d'exploitation n'est plus assurée, les comptes annuels doivent être établis sur la base de la valeur liquidative»(CNCC, 1996, p.4). Ces principes supposent que la comptabilité délivre une information prédictive.

En comptabilité française, si le risque de défaillance est avéré (continuité incertaine), il faudra préciser en annexe les hypothèses retenues et les facteurs qui expliquent le maintien du principe de continuité. Si la continuité n'est plus assurée, les principes comptables de base ne peuvent plus être appliqués puisque l'établissement des comptes en valeur liquidatives implique une atténuation du principe de prudence ; la permanence des méthodes est de facto abandonnée. Comme le rappelle Colasse (2001, p.59), la continuité d'exploitation «légitime en particulier la ventilation des charges et des produits entre les différents exercices de la vie 
des entreprises ». La cessation des paiements, stade ultime de la rupture dans la continuité d'exploitation, demeure très délicate à prédire et de nombreux groupes et sociétés présentent leurs comptes selon le principe de continuité tout en étant virtuellement en faillite, car en recherche active de fonds, et avec un dépôt de bilan très proche de la date de clôture du dernier exercice (CNCC, 1996).

Ainsi l'annexe devient le support primordial pour l'utilisateur des comptes qui souhaite estimer le risque de défaillance. A ce titre, les normes américaines et internationales privilégient fortement ces modalités de communication.

\subsubsection{Le cadre comptable américain (US GAAPS) : une approche prédictive du "going} concern »

Ce cadre comptable américain fait très peu référence à la notion de continuité d'exploitation. Le SFAC $5^{8}$ qui présente les concepts fondamentaux d'évaluation et de comptabilisation du cadre comptable américain propose la définition suivante de la continuité d'exploitation («Going Concern»): «une entité est présumée, sauf avis contraire, poursuivre son activité dans une échéance suffisamment lointaine pour poursuivre l'ensemble de ses engagements. L'établissement des états financiers repose donc sur le principe de continuité ». En outre, à la différence du cadre français, les objectifs des états financiers ne sont pas nécessairement modifiés si l'entreprise passe du stade de continuité à celui de valeur liquidative. L'annexe prend une place importante car c'est la pertinence et le caractère prédictif de l'information qui doit révéler le risque de défaillance et la cessation des paiements. Ainsi, le cadre américain confirme l'importance donnée à l'annexe pour conférer une dimension prédictive à l'information comptable (Mutchler et Smith, 1984). Par ailleurs, les conventions d'évaluation reposent sur le concept de juste valeur. Les normes FAS 121 et FAS 114 relatifs aux actifs préconisent un traitement des dépréciations au moyen de méthodes 
d'actualisation de flux de trésorerie futurs. Les FAS 107, FAS 115 et FAS 133 relatifs aux instruments financiers introduisent très clairement des valorisations de clôture à la valeur de marché. Ces valorisations ont davantage une portée informationnelle prédictive que le coût historique qui demeure un constat du passé. Dans le prolongement de ces postulats, le cadre conceptuel IFRS corrobore ces évolutions en affirmant que la comptabilité a pour objectif d'être une aide à la décision des actionnaires. En ce sens le caractère prédictif de la comptabilité est renforcé.

\subsubsection{Le cadre comptable international (IAS / IFRS): une perspective résolument} économique de l'activité de l'entreprise

La continuité d'exploitation est énoncée dans le cadre conceptuel ${ }^{9}$ : « Les états financiers sont normalement préparés selon l'hypothèse qu'une entreprise est en situation de continuité d'exploitation et poursuivra ses activités dans un avenir prévisible. Ainsi, il est supposé que l'entreprise n'a ni l'intention, ni la nécessité de mettre fin à ses activités, ni de réduire de façon importante la taille de ses activités. S'il existe une telle intention ou une telle nécessité, les états financiers peuvent devoir être préparés sur une base différente, et, s'il en est ainsi, la base utilisée doit être indiquée». Cette définition demeure assez proche des définitions données par le PCG et par les US GAAP. Elle doit être comprise comme la possibilité pour une entreprise d'évaluer ses actifs à une valeur supérieure à une valeur de liquidation. Ce principe, sous-jacent à l'établissement des comptes en normes IFRS, renvoie sur l'application de certaines caractéristiques qualitatives des états financiers comme l'intelligibilité (cadre IFRS, § 25) et la pertinence (Cadre IFRS, § 26, 27 et 28). Ainsi une information ne peut être exclue des états financiers en raison de sa complexité (intelligibilité) et l'information financière doit influencer les décisions économiques des utilisateurs des comptes (pertinence). 
Les incertitudes pesant sur la continuité d'exploitation doivent être indiquées et en cas de cessation des paiements l'entreprise doit préciser la base sur laquelle les comptes ont été arrêtés. En conséquence l'information en annexe est prédominante pour informer les tiers sur les risques de défaillance.

Comme le cadre comptable IFRS, tous les cadres comptables ont pour objectif de fixer la prédiction de la défaillance des entreprises en obligeant celle-ci à respecter le principe de continuité d'exploitation. Il s'agit pour le normalisateur de pousser les entreprises et leurs auditeurs à prédire les difficultés financières de l'entreprise sur la base des éléments comptables les plus récents.

Au regard de l'évolution normative des 30 dernières années, il n'est pas interdit de penser que les normalisateurs ont préféré entretenir un certain flou dans la définition du principe de continuité d'exploitation pour faire émerger des valorisations de clôture qui privilégient des calculs actuariels reposant sur des hypothèses futures. Cette évolution n'est pas absolue et l'abandon à la fin des années 90 du projet «Full Fair Value » en est le très bon exemple. Tous les postes comptables ne sont pas impactés par ces valorisations prédictives et le coût historique demeure la valorisation de référence pour les actifs d'investissement, les stocks et les créances. Néanmoins comme le rappellent Richard et Colette (2000, pp.56-7): "l'hypothèse de la non-continuité (mort) n'empêche pas que dans les phases de difficultés de l'entreprise il soit intéressant de connaître à la fois la valeur de marché des actifs (grâce à une comptabilité statique) et la mauvaise performance de l'entreprise (grâce à une comptabilité dynamique $\left.{ }^{10}\right)$. En résumé, une fois qu'on a émis l'hypothèse de continuité ou de 
non continuité d'exploitation, on a certes donné une information fondamentale, mais on a en aucune manière indiqué un choix de type de comptabilité ».

L'évolution de l'approche prédictive de la comptabilité est donc partielle car présenter l'ensemble du bilan en valeur de marché n'est probablement pas plus prédictif que de le présenter intégralement au coût historique. L'approche prédictive a donc deux conséquences majeures. D'une part, les entreprises, sur une base forcément subjective (comptabilité d'intention), développent des modèles actuariels pour certains de leurs actifs et dettes et d'autre part, ces mêmes entreprises sont contraintes de détailler leur information qualitative en annexe pour expliquer aux tiers les hypothèses appliquées pour, en définitive, respecter la comparabilité des états financiers. L'évolution de la comptabilité vers un modèle prédictif repose sur des valorisations actuarielles qui sont elles-mêmes indissociables de l'information en annexe.

Les recherches académiques confirment cet axe de réflexion puisque les nombreuses recherches antérieures s'inscrivent dans une approche prédictive et positive de la comptabilité (White et al., 2002).

\subsection{Revue de littérature}

L'utilisation de l'information financière destinée à prédire le risque de défaillance d'une entreprise a fait l'objet de nombreuses recherches principalement aux Etats-Unis. En outre, depuis les années 60, de nombreux auteurs se sont intéressés à la prédiction de la faillite des entreprises.

\subsubsection{Information financière et risque de défaillance}

L'information financière sur le risque de défaillance est une thématique qui couvre en réalité deux grands axes de recherche, l'offre et la demande d'information, avec d'une part, l'étude 
de l'offre d'information en situation de risque et d'autre part, l'étude de la demande en observant les travaux effectuée par les utilisateurs pour identifier le risque de non continuité.

\section{- Offre d'informations comptables et défaillance}

La plupart des recherches qui établissent un lien entre comptabilité et défaillance sont axées sur l'étude de l'offre d'information. Ainsi, Schwartz (1982), Opler et Titman (1994) et De Angelo et al. (1994) ont analysé les choix comptables, souvent opportunistes, pris par des entreprises soumises à un risque élevé de non continuité.

L'utilisateur des comptes est donc en mesure de s'interroger sur la crédibilité de l'information publiée (Vilanova, 1997): d'une part, Opler et Titman (1994) montrent qu'un dirigeant d'entreprise est tenté de dissimuler aux tiers les difficultés financières de son entreprise, d'autre part, Teller (1998) s'interroge sur la pertinence du modèle comptable dès lors que l'entreprise connaît un risque de défaillance significatif (rupture de la continuité d'exploitation).

L'offre d'information n'est donc pas forcément adaptée à la demande d'information nécessaire à l'analyse du risque de non continuité. Saboly (2001, p.71) qui rappelle la nécessité de recourir à des informations non comptables, précise : «En effet, la comptabilité fournit une représentation de la réalité construite selon certaines contraintes et conventions. Un des biais de cette représentation est qu'elle n'englobe pas la totalité du réel: la comptabilité sélectionne certains faits en fonction de son propre ordre de référence et dans la mesure où ils peuvent être traduits en unités monétaires ».

En conséquence, les utilisateurs des états financiers peuvent être amenés à utiliser des modèles financiers nécessaires pour prédire les défaillances en amont de l'apparition du risque de non continuité. 


\section{- Demande d'informations comptables et défaillance}

Les recherches en comptabilité et finance qui traitent de l'estimation du risque de continuité d'exploitation reposent très souvent sur des ratios financiers. Beaver (1966) propose des méthodes d'analyse financière aujourd'hui assez répandues en pratique. Sur un angle plus statistique, Koh et Killough (1990) proposent des analyses discriminantes «pas à pas », Mensah (1983), quant à lui, s'appuie sur des analyses en composante principale.

Ainsi, il apparait raisonnable de considérer que l'estimation du risque de non continuité consiste à réaliser une appréciation analytique et comparative de certains postes comptables sur plusieurs périodes. En outre, les ratios détectant ce risque varient fortement d'un secteur d'activité à un autre d'après Foster (1986). Néanmoins, un ratio ne peut être interprété qu'en examinant la cohérence de son évolution dans le temps, évolution qui pourra utilement aider l'entreprise à extrapoler dans l'avenir l'évolution de ce même ratio.

Compte tenu de l'évolution du cadre comptable et afin de préparer un cadre d'analyse efficace, la théorie prédictive de la comptabilité permet de dégager des ratios pertinents en termes d'appréciation du risque de défaillance.

\subsubsection{Information financière et approche prédictive: modèle comptable vs. approche optionnelle}

Le travail principal incombant à tout analyste crédit ou auditeur financier externe est d'évaluer la probabilité qu'une entreprise soit confrontée, sur un horizon plus ou moins éloigné, à une situation de détresse financière, ou autrement dit, qu'elle ne soit plus en mesure d'honorer raisonnablement ses dettes contractées.

Les auteurs aussi bien que les professionnels s'accordent volontiers à reconnaitre que le travail d'appréciation de la détresse financière prévisionnelle d'une entreprise est, bien souvent, complexe, fastidieux et, largement subjectif, notamment en raison des multiples 
étapes qu'il implique, étapes qui peuvent regrouper tant de l'analyse stratégique, que de l'analyse «traditionnelle » comptable et financière ou encore de l'analyse prospective. Les modèles prévisionnels de risque de défaut se présentent souvent, alors, comme une solution alternative bien peu onéreuse en comparaison de l'implémentation d'une telle analyse « avancée » de crédit.

Dans un article précurseur, Beaver (1968) établit un lien entre qualité de l'information et sa capacité prédictive. Ainsi la qualité de l'information comptable repose sur son aptitude à fournir une information utile pour les décisions futures de l'investisseur. La prédiction est très utilisée pour détecter la défaillance afin d'éviter le dépôt de bilan de l'entité. Le modèle de Beaver (1966) fondé sur une variable à la fois a montré la pertinence de deux ratios (Autofinancement / Dettes totales ; Profit net / Actif total) pour détecter les risques de non continuité. Les résultats probants du travail de Beaver (1968) alliée à une volonté de minimiser les coûts d'analyse de crédit expliquent la prolifération des modèles prospectifs comptables au cours des 30 dernières années dans les pratiques d'analyse de crédit. Ces modèles, comme leurs noms l'indiquent, présentent la singularité de ne reposer que sur des variables comptables issues directement des états financiers d'une entreprise et sont assimilables, à de nombreux égards, aux modèles de «debt rating ». Le modèle Zeta d'Altman (1968) reste, sans aucun doute, l'un des plus utilisés par les praticiens.

L'estimation du risque de faillite d'une entreprise industrielle ou commerciale s'effectue ainsi, traditionnellement, à l'aide d'indicateurs fondés sur des données comptables et financières issus eux-mêmes des états financiers. Cette méthode dite «traditionnelle » est souvent qualifiée d'inappropriée par les auteurs et ce, pour trois principales raisons. En premier lieu, la probabilité de défaillance est, par essence même, une mesure prospective dont la validité se jugera dans les prises de décisions et activités futures de l'entreprise. A ce titre, les états financiers en présentant des données traduisant les performances sociétales passées, 
s'avèrent, trop souvent, peu informatifs quant à l'avenir d'une société et proposent, de facto, des données faiblement corrélées au risque de faillite. En second lieu, les états financiers sont établis conformément au principe de continuité d'exploitation, principe dont l'hypothèse sousjacente majeure est que l'entreprise ne fera pas faillite durant la période financière à venir. En ce sens, leur capacité à apprécier, de manière précise et raisonnable, la probabilité de faillite d'une entreprise en est d'autant affaiblie. Enfin, l'application systématique aux transactions comptables du principe de prudence a pour conséquence directe de sous-évaluer, de manière récurrente, la valeur des actifs au regard de leurs valeurs de marché, le cas des actifs incorporels restant un exemple illustratif classique. Ces biais de valorisation, à l'impact négatif, dans l'évaluation de ces actifs induisent des leviers comptables surestimés.

Une autre source importante d'inanité des modèles comptables de faillite est leur incapacité inhérente à tenir compte de la volatilité des actifs. Cette variable s'avère cruciale dans les prédictions du risque de défaut, en raison de sa capacité à capturer l'information permettant de déterminer si la valeur des actifs d'une entreprise déclinera jusqu'au point de ne plus couvrir la valeur des dettes contractées. Ainsi, toute chose égale par ailleurs, la probabilité de défaillance peut être considérée comme étant positivement et significativement corrélée avec la volatilité des actifs. Par voie de conséquence, deux entreprises avec un levier d'endettement identique peuvent, néanmoins, présenter des risques de défaut distincts, du simple fait de la différence entre leurs volatilités intrinsèques. A ce titre, les auteurs et les praticiens considèrent que la volatilité est bien trop souvent exclue des modèles de prédiction de faillite (ex. les modèles du score d'Ohlson (1980) et d'Altman (1968)).

Une solution potentielle pour pallier à ce déficit serait d'intégrer des mesures de volatilité de marché dans les modèles prévisionnels. En effet, les marchés financiers peuvent être logiquement perçus comme une source d'information alternative et potentiellement supérieure 
à celle résultant de l'utilisation des variables comptables ( $c f$. les travaux du Professeur Beaver datant du début des années 1960). Cependant, la difficulté majeure rencontrée tant par les chercheurs que par les praticiens est d'extraire de la multitude d'informations fournies par le marché celles qui s’avèrent pertinentes pour le calcul des risques de défaut.

Dès le début des années 1990, les modèles de valorisation d'options tels que formulés par Francis Black \& Myron Scholes, en 1973, et Robert Merton, en 1974, ont été proposés comme point de départ naturel à la réflexion. Leur application à l'analyse de crédit reste simple. Il suffit, en effet, de considérer, dans ces modèles, que la valeur des titres de l'entreprise peut être assimilée à une option d'achat sur la valeur des actifs de l'entreprise. Quand la valeur des actifs est inférieure à la valeur nominale des dettes, c'est-à-dire au prix d'exercice, l'option d'achat n'est alors pas exercée et le risque de défaut de l'entreprise est naturellement reporté sur les créanciers. Le principal avantage lié à l'utilisation des modèles de valorisation d'options pour apprécier le risque de défaut est qu'ils fournissent des relations stables et pertinentes concernant les déterminants théoriques des risques de non continuité ainsi que la structure de modélisation nécessaire pour extraire les informations pertinentes des prix de marché. Ce bénéfice indéniable vient néanmoins en contre-balancement de deux inconvénients majeurs. Tout d'abord, les hypothèses simplificatrices des modèles de Black \& Scholes et de Merton (ci-après BSM) sont loin d'être toujours observées et a fortiori valables en pratique. De fait, ces hypothèses introduisent des erreurs dans l'estimation du risque de défaut. Une autre source potentielle d'erreur serait de considérer que le marché financier traduit dans les prix des titres toutes les informations publiquement disponibles à un instant donné. De nombreuses études empiriques ont déjà montré que le marché ne faisait pas refléter dans le cours des actions toutes les informations contenues dans les états financiers. 
Néanmoins, de récentes recherches sur le sujet viennent d'apporter la preuve que les modèles de «pricing d'options » présentent des pouvoirs explicatifs nettement supérieurs aux modèles comptables traditionnels de risque de faillite. Par exemple, en analysant un échantillon de 864 faillites de sociétés américaines observées sur la période 1971-1999 mis en regard d'un panel de contrôle d'environ 100.000 entreprises, Vassalou et Xing (2004) observent que le modèle BSM permet d'expliquer, en médiane, plus de 92\% des défaillances ayant eu lieu sans pour autant conférer un risque de défaut significatif aux entreprises appartenant au groupe de contrôle. Ces résultats surpassent de loin ceux générés par les modèles du score Zeta et d'Ohlson (1980), modèles qui imputent, communément, un risque de défaut significatif aux entreprises financièrement «saines ». Néanmoins, en moyenne, les résultats du modèle BSM restent encore peu satisfaisants. Deux limites peuvent, cependant, expliquer ce dernier constat : tout d'abord, la difficulté de différencier les entreprises rentrant juridiquement dans un processus de défaillance et celles étant en situation de non continuité d'exploitation mais faisant l'objet d'un rachat ou d'une reprise ; d'autre part, la non observation de la normalité des rendements de titre, hypothèse sous-jacente au modèle BSM.

Aux vues de ces résultats concluants ( $c f$. Hillegeist et al., 2004) et en gardant à l'esprit les imperfections des modèles prévisionnels comptables, l'application du modèle BSM à l'analyse de crédit reste, sans nul doute, une voie complémentaire à celle des modèles comptables.

\subsection{Question de recherche}

Si le principe de continuité d'exploitation est conceptuellement faible dans les modèles comptables $^{11}$, son estimation est une donnée importante de la prise de décision de l'investisseur. Même si la détection du risque de défaillance est délicate, de nombreux auteurs depuis Altman (1968) ont cherché à établir un lien entre information financière et 
évaluation du risque de défaillance. Dans le prolongement de ces études et sur la base des modèles économétriques présentés ci-avant, nous avons cherché à montrer les différences d'estimation du risque de défaillance ou de non continuité susceptibles d'être constatées entre l'application du référentiel français et l'application du référentiel IFRS. L'étude est donc réalisée sur la base d'une analyse comparative de l'information financière en normes Françaises et en normes IFRS, telles que publiées par les groupes cotés pour une même période. Ainsi, peut-on considérer que le changement de référentiel et donc le passage d'un système statique à un système actuariel, donc plus prédictif et privilégiant l'information narrative, entraîne un changement significatif sur l'estimation du risque de défaillance ?

La prochaine section discute les principaux modèles développés dans notre analyse et examine les impacts potentiels du passage aux normes IFRS sur les principaux modèles de risque de défaut. Cette première analyse permet de donner les premiers principes fondamentaux de notre approche d'investigation.

\section{Descriptif et analyse des principaux modèles prédictifs de défaut}

Dans la littérature académique, le risque de défaillance, c'est-à-dire le risque que l'exploitation de l'entreprise ne puisse être continuée, a souvent été appréhendé par modélisation. Tel que discuté précédemment, les différentes méthodes académiques proposées pourraient être regroupées en deux catégories suivant qu'ils adoptent (1) une approche d'économétrie linéaire ou (2) une approche optionnelle, i.e. fondée sur les modèles de valorisations d'option. 


\subsection{Les modèles basés sur l'information comptable}

Le principe de ces méthodes traditionnelles se fonde sur des régressions de type logit dont l'objet est de déterminer si une entreprise présente un risque significatif de défaillance. Leur formulation est basée sur le principe suivant : Le modèle décrit par l'équation (1) établit qu'une entreprise sera en rupture de continuité d'exploitation $(\mathrm{GC}=0)$ lorsque une variable sous-jacente $\mathrm{GC}^{*}$ excède un seuil $k$ donné (k étant dans la plupart des modèles égal à zéro).

$$
\begin{aligned}
& P(G C=0)=P\left(G C^{*}>k\right) \\
& G C^{*}=\lambda_{0}+\sum_{k=1}^{n} \lambda_{k} . V A R_{k}+\varepsilon
\end{aligned}
$$

avec

$P($.$) , la probabilité de (.) ;$

$G C=0$ dans le cas d'une rupture de la continuité d'exploitation et 1 autrement ;

$V A R$, les variables d'influence de la continuité d'exploitation ;

$\lambda_{i}$, le coefficient (ou loading) de la variable d'influence $i$ dans le modèle ;

$u$, un terme résiduel normalement distribué.

Les coefficients $\lambda_{i}$ sont déterminés à partir d'échantillons représentatifs et testés sur un groupe test. L'objectif de cette session est de discuter l'impact potentiel du changement de référentiel sur les coefficients des principales variables d'influence. Le tableau 1 présente les principaux modèles variables d'influence les plus communément considérées lors de la modélisation ainsi que les modèles qui les utilisent :

[INSERER TABLEAU 1] 
Le tableau 1 souligne que ces modèles font appel, de manière exhaustive, à des variables construites à partir des données comptables suivantes :

- $\quad$ Total des actifs

- Total des actifs courants

- Total des passifs courants

- BFR (tel que mesuré par actifs courants - passifs courants)

- Chiffre d'affaires

- Résultat net

- Résultats mis en réserves

- $\quad$ EBIT (ou EBE)

- Total des dettes (court et long-terme)

Néanmoins, comme le soulignent de nombreux auteurs (ex. Vassalou et Xing (2004), Koh (1991)), les modèles comptables font preuve de lacunes significative ne permettant de baser une prédiction de défaut d'une entreprise uniquement sur une telle approche. Dans cette optique, la littérature appuyée sur les travaux fondateurs de Merton (1974) a proposé une seconde approche d'appréhension du risque de défaut basée sur les modèles couramment utilisés pour valoriser une option ou modèles optionnels.

\subsection{Les modèles optionnels}

Le modèle de Merton (1974) émet l'hypothèse que la valeur des capitaux propres d'une entreprise peut être assimilée à une option de type européen dont le sous-jacent serait les actifs de l'entreprise. Le fondement de cette approche réside dans le constat que les actionnaires en tant qu'ayants-droit résiduels des actifs de l'entreprise (après les créanciers) supportent une responsabilité limitée directement proportionnelle à leurs mises de fonds initiales, i.e. le nombre d'actions achetées. De cette façon, les fonds leur revenant sont 
identiques à ceux d'une option de vente. Le prix d'exercice de l'option n'est alors que le niveau d'endettement de l'entreprise. En effet, si la valeur des actifs de l'entreprise se trouve être inférieure au montant de ses dettes, la valeur des capitaux propres de l'entreprise est alors nulle et la défaillance est avérée. En s'appuyant sur le modèle de valorisation de Black et Scholes (1973), il est alors possible de montrer que :

$$
V_{C P}=V_{A} N\left(d_{1}\right)-K e^{-r t} N\left(d_{1}-\sigma_{A} \sqrt{T}\right)
$$

avec $d_{1}=\frac{\ln \left(V_{A} / K\right)+\left(r+0,5 \cdot \sigma_{A}^{2}\right) \cdot T}{\sigma_{A} \sqrt{T}}$ et $\mathrm{V}_{\mathrm{A}}$ la valeur de marché des actifs, $\mathrm{K}$ la valeur comptable des dettes à la date de maturité $\mathrm{T}, \mathrm{r}$ le taux sans risque, $\sigma_{\mathrm{A}}$ l'écart-type de $\mathrm{V}_{\mathrm{A}}$ et $\mathrm{N}($.$) la fonction cumulative de densité de la loi normale standard.$

Le modèle de Merton (1974) s'appuie sur une hypothèse de normalité de la croissance de la valeur des marchés des actifs de l'entreprise. La valeur de ces actifs $\mathrm{V}_{\mathrm{A}}$ est alors, à tout instant $\mathrm{t}$, égale à :

$$
\ln \left(V_{A, T}\right) \square \mathrm{N}\left(\ln \left(V_{A, 0}\right)+\left(\mu_{A}-0,5 \cdot \sigma_{A}^{2}\right) T, \sigma_{A}^{2} \cdot T\right)
$$

De cette hypothèse, il est possible de déduire la probabilité que $\mathrm{V}_{\mathrm{A}}$ soit inférieure à $\mathrm{K}$, i.e. la probabilité de défaut (PDEF), est égale à :

$$
P D E F=1-\mathrm{N}\left(\frac{\ln \left(V_{A} / K\right)+\left(\mu-0,5 . \sigma_{A}^{2}\right) T}{\sigma_{A} \sqrt{T}}\right)
$$

En utilisant l'approche par les «greeks », il est possible de montrer que la sensibilité du modèle à la valeur comptable des dettes peut être exprimée comme :

$$
\frac{d V_{C P}}{d K}=-e^{-r t} N\left(d_{1}-\sigma_{A} \sqrt{T}\right)+e^{-r t} N^{\prime}\left(d_{1}-\sigma_{A} \sqrt{T}\right) * \frac{1}{\sigma_{A} \sqrt{T}}
$$




$$
\frac{d P D E F}{d K}=\mathrm{N}^{\prime}\left(\frac{\ln \left(V_{A} / K\right)+\left(\mu-0,5 . \sigma_{A}^{2}\right) T}{\sigma_{A} \sqrt{T}}\right) * \frac{(-1)}{K \sigma_{A} \sqrt{T}}
$$

Ces deux relations permettent donc, en théorie, de jauger l'impact de la variation des dettes liées à la transition aux normes IAS / IFRS. Ils constituent notre base de test empirique relativement à l'impact de la transition IAS / IFRS sur l'estimation du risque du défaut à partir du modèle de Merton (1974).

La section suivante présente les résultats de l'étude empirique et leurs analyses. La méthode d'échantillonnage est décrite en premier lieu.

\section{Etude exploratoire sur le marché français}

\subsection{Echantillonnage}

Notre échantillon de départ est constitué de l'ensemble des sociétés cotées composant l'indice SBF $120^{12}$ à la date du 31 décembre 2004, veille du passage aux normes IFRS. Les variables nécessaires à la mise en place des modèles de risque de défaut discutés précédemment sont collectées manuellement à partir des rapports annuels 2004 et 2005 des sociétés échantillonnées. Notre objectif est d'obtenir ces variables établies selon les normes internationales IAS / IFRS et les normes françaises (French GAAPs) en vue de permettre une analyse, ceteris paribus, de l'impact du passage aux normes IFRS sur ces procédures d'évaluation de risque. De manière plus détaillée, nous collectons les données au 31 décembre 2004 établies selon les French GAAPs à partir des rapports annuels 2004 et recueillons ces mêmes données en référentiel IFRS dans les rapports annuels $2005^{13}$. Ainsi les données comptables 2004 IFRS sont celles qui ont été publiées dans les comptes comparatifs 2005. En effet, la norme IFRS 1 (§ 8) suppose que les premiers états financiers IFRS soient 
publiés comme si les normes internationales avaient été appliquées depuis l'origine de l'entité. En conséquence, les ajustements comptables rétrospectifs sont enregistrés en capitaux propres d'ouverture au $1^{\mathrm{er}}$ janvier 2004 sauf pour les applications exemptés sur option de cette méthode rétrospective (IFRS $1 \S 13$ ).

De cet échantillon, sont exclues (1) toutes les sociétés financières, i.e. celles présentant un code sectoriel New FTSE / DJ Industry Classification Benchmark (ICB) ${ }^{14}$ compris entre 8000 et 8999 , en raison du particularisme de leurs règles comptables et financières sectorielles ainsi que (2) toutes les sociétés dont les variables comptables et financières nécessaires à l'analyse ne sont pas directement disponibles à partir des rapports annuels. Un échantillon final d'analyse de 90 sociétés cotées est ainsi obtenu. Le tableau 1 présente une ventilation sectorielle de cet échantillon en utilisant le niveau 2 de la classification ICB. De ce tableau, il convient de noter que deux groupes sectoriels sont surreprésentés dans l'échantillon: les «Médias » (5500) et les «Biens et services industriels » (2700) qui représentent respectivement $12 \%$ et $24 \%$ des observations. Afin de pallier l'éventualité que cette surreprésentativité puisse biaiser nos résultats statistiques en raison d'éventuelles spécificités sectorielles liées à ces deux secteurs, l'impact de ces groupes sur notre analyse a donné lieu, dans notre étude, à des tests de sensibilité dont les résultats ne se sont pas avérés concluants.

[INSERER TABLEAU 2]

\subsection{Résultats empiriques}

\subsubsection{Statistiques univariées}

Les statistiques relatives aux agrégats comptables nécessaires à la construction des variables d'analyse des modèles comptables de risque de défaut sont présentées dans le tableau 3. Les 
variables comptables sont toutes exprimées en millions d'euros. Le tableau 3 souligne quatre points intéressants : (1) la moyenne (médiane) du chiffre d'affaires est respectivement de 14256 (7 994) en normes françaises et de 13758 (7 787) en normes IAS / IFRS ce qui laisse supposer que le référentiel international est moins flexible pour reconnaître les transactions de vente ; (2) le besoin en fonds de roulement est nettement inférieur en référentiel IAS / IFRS. Les normes internationales présentent une classification des actifs courants moins permissive et des passifs courants plus strictes que le référentiel français ; (3) en moyenne (médiane), le résultat net de la période en IFRS est de 765,29 $(269,50)$ contre seulement 561,22 $(215,10)$ en French GAAPs. Ce résultat souligne que les sociétés du SBF 120 ont profité du passage aux normes IFRS pour constater davantage de résultat sur le bilan d'ouverture suivant les dispositions de la norme IFRS 1. Ces gains constatés en plus en référentiel IFRS semblent néanmoins être exceptionnels puisque l'EBE est relativement identique en IFRS (1 309 en moyenne et 519 en médiane) à celui constaté en French GAAPs (1 315 en moyenne et 530 en médiane) ; (4) le référentiel IFRS semble faciliter la reconnaissance des dettes financières à court terme puisqu'elles passent d'un montant en moyenne (médiane) de 794 (7,50) en French GAAPs à un montant de 1683 (383) en IAS / IFRS. Ce dernier résultat est confirmé par le test bilatéral de Mann-Whitney-Wilcoxon qui montre que les dettes financières à court terme sont différentes, de manière significative, entre l'échantillon en French GAAPs et le même échantillon divulguant en normes IAS / IFRS.

[INSERER TABLEAU 3]

\subsubsection{Analyse de l'impact IFRS sur l'estimation du risque de défaut par les modèles}

\section{- Modèles comptables prédictifs}


Les panels $\mathrm{A}$ et $\mathrm{B}$ du tableau 4 présentent respectivement les statistiques univariées et bivariées pour les variables d'analyse retenues par les modèles d'Altman (1968), Ohlson (1980) et Zmijewski (1984).

Le panel A présente les variations liées à l'application des normes IAS / IFRS pour chaque variable d'intérêt. Les différents impacts liés à la transition aux normes IFRS sur les trois modèles comptables de risque de défaut peuvent être résumés par les équations suivantes :

(1) Modèle d'Altman (1968) :

$\frac{\mathrm{dGC}_{\mathrm{Altman}}^{*}}{\mathrm{dIAS}}=-0,34 \cdot \lambda_{1} \cdot \frac{\mathrm{BFR}}{\text { Actifs }}-0,04 \cdot \lambda_{2} \cdot \frac{\mathrm{CA}}{\text { Actifs }}-0,26 \cdot \lambda_{2} \cdot \frac{\mathrm{EBE}}{\text { Actifs }}-0,07 \cdot \lambda_{2} \cdot \frac{\text { Capitalisation }}{\text { Dettes }}+\varepsilon$

(2) Modèle d'Ohlson (1980) :

$$
\begin{aligned}
\frac{\mathrm{dGC}_{\text {Ohlson }}^{*}}{\mathrm{dIAS}}=0, & 15 \cdot \beta_{1} \cdot \log (\text { Actifs })-0,34 \cdot \beta_{2} \cdot \frac{\mathrm{BFR}}{\text { Actifs }}+0,31 \cdot \beta_{3} \cdot \frac{\text { Passifs Courants }}{\text { Actifs Courants }} \\
& +1,05 \cdot \beta_{4} \cdot \frac{\mathrm{RN}}{\text { Actifs }}+0,23 \cdot \beta_{5} \cdot \text { VariationRésultat }+0,01 \cdot \beta_{6} \cdot \frac{\text { Passifs }}{\text { Actifs }}+\varepsilon
\end{aligned}
$$

\section{(3) Modèle de Zmijewski (1984) :}

$\frac{\mathrm{dGC}_{\text {Zmijewski }}^{*}}{\mathrm{dIAS}}=3,23 \cdot \beta_{1} \cdot \frac{\text { Actifs Courants }}{\text { Passifs Courants }}+1,05 \cdot \beta_{2} \cdot \frac{\text { Résultat net }}{\text { Actifs }}+0,09 \cdot \beta_{3} \cdot \frac{\text { Dettes }}{\text { Actifs }}+\varepsilon$

L'équation (1) montre que la transition aux normes IFRS tend, ceteris paribus, à augmenter la probabilité de défaut de l'entreprise puisque l'on observe une augmentation de GC* ce qui implique une augmentation de $P\left(G C^{*}>k\right)$. Si établi à partir de données French GAAPs, le modèle d'Altman (1968) semble donc défavoriser, en moyenne, les entreprises passant aux normes IFRS dans notre échantillon. 
Le modèle d'Ohlson (1980) (équation 2) en revanche semble favoriser, en moyenne, la transition aux normes IFRS puisqu'il paraît être impacté positivement par le passage au référentiel international (en considérant que l'impact négatif du BFR est annihilé par les autres variables). De même, le modèle de Zmijewski (1984) voit le risque de défaut d'une entreprise diminuer en moyenne à l'occasion du passage aux normes IFRS.

En résumé, ceteris paribus, à l'occasion du passage aux normes IAS / IFRS, le risque de défaut dans notre échantillon diminue dans le modèle d'Ohlson (1980) et de Zmijewski (1984) et tend à augmenter dans le modèle d'Altman (1968).

\section{[INSERER TABLEAU 4]}

Le panel B du tableau 4 présente les coefficients de corrélation de Pearson et Spearman (méthode des rangs) pour toutes les variables indépendantes des modèles comptables de risque de défaut. Les statistiques sont présentées par référentiel normatif. Pour le modèle d'Altman (1968), les résultats indiquent que les variables ne sont pas plus auto-corrélées en normes françaises qu'en référentiel international excepté pour la variable LIQ2 et PROF2 (leur corrélation est non significative en French GAAPs et significative aux seuils conventionnels en normes IAS / IFRS). Elle pourrait donc induire un biais d'auto-corrélation dans le modèle d'Altman (1968) lorsqu'utilisé en référentiel international. Concernant le modèle d'Ohlson (1980), les variables du modèle semblent plus corrélées, dans notre échantillon, en référentiel IAS / IFRS qu'en normes françaises (ex. LIQ3 et TAILLE, LIQ3 et PROF1, PROF1 et TAILLE, PROF1 et GEAR2, PROF3 et LIQ3). Par conséquent, sa mise en place en référentiel international semble nécessiter des précautions supplémentaires en vue de pallier aux éventuels problématiques d'auto-corrélation des variables indépendantes du 
modèle. Cette dernière remarque reste valable dans le cadre du modèle de Zmijewski (1984) puisque les variables PROF1 et GEAR3, et PROF1 et LIQ3 présentent une corrélation significative dans l'échantillon IAS / IFRS. 


\section{- Modèles optionnels}

Le tableau 5 présente les résultats de l'impact de la transition aux normes IFRS sur le modèle optionnel de risque de défaut de Merton (1974) adapté par Vassalou et Xing (2004) suivant différents scenarii liés à la volatilité et à la croissance estimée des actifs de l'entreprise. Le panel A, B et C présentent respectivement la sensibilité de la valeur des capitaux propres, de la probabilité de défaut et la variation de la probabilité de défaut liées à un changement du montant des passifs (hors capitaux propres) lors de la transition aux normes IAS / IFRS. A l'instar de Vassalou et Xing (2004) et Hillegeist et al. (2004), une maturité et un taux sans risque de 1 an ont été considérés en vue de l'estimation de la probabilité de défaut de chaque entreprise échantillonnée. Le prix d'exercice de l'option est égal au montant du total des dettes de l'entreprise ${ }^{15}$.

Le panel $\mathrm{C}$ indique qu'en moyenne le passage aux normes IFRS implique une diminution du risque de défaut par l'approche optionnelle entre $0,09 \%$ et $0,32 \%$ suivant les scenarii. Néanmoins, le risque de défaut semble invariant lorsque la médiane est considérée.

En résumé, les normes IFRS semblent avoir permis, dans notre échantillon, de diminuer en moyenne le risque de défaut tel qu'estimé à partir du modèle optionnel de Merton (1974) et des modèles comptables d'Ohlson (1980) et Zmijewski (1984). En revanche, le modèle d'Altman (1968), suite à la transition IFRS, augmente en moyenne le risque de défaut des entreprises échantillonnées.

[INSERER TABLEAU 5] 


\section{Conclusion}

A l'aide des informations comparatives IFRS / French GAAPs divulguées dans les rapports annuels 2005 d'entreprises industrielles françaises du SBF 120, cette étude analyse si la transition des normes comptables françaises vers le référentiel IFRS impacte de manière significative l'estimation du risque de défaut issue des modèles traditionnels de type comptable et optionnel. Les preuves empiriques rapportées dans cette étude montrent que, toutes choses égales par ailleurs, l'estimation du risque de défaut diminue de manière significative du seul fait du passage aux normes IFRS. Plus précisément, nos principaux résultats s'articulent autour de trois points. En premier lieu, nous constatons que l'application des normes IFRS favorise une reconnaissance des dettes financières dans les comptes consolidées des sociétés cotées du SBF 120 comprises dans notre échantillon et présente une classification des actifs courants moins permissive et des passifs courants plus strictes que le référentiel français. Deuxièmement, nos résultats démontrent qu'à l'occasion du passage aux normes IAS / IFRS, le risque de défaut dans notre échantillon diminue dans le modèle d'Ohlson (1980) et de Zmijewski (1984) et tend à augmenter dans le modèle d'Altman (1968). Enfin, les normes IFRS semblent avoir permis, dans notre échantillon, de diminuer en moyenne le risque de défaut tel qu'estimé à partir du modèle optionnel de Merton (1974) et de Vassalou et Xing (2004).

Notre approche est cependant sujette à quatre principales critiques. Premièrement, nous ne faisons que tester l'impact du passage aux normes IFRS sur les coefficients de régression et les sensibilités liées aux modèles optionnels. Pour compléter ce travail d'analyse, il conviendrait, pour constater si les modèles de "going-concern" sont à remettre en question en référentiel IFRS, de réestimer l'ensemble des coefficients à partir d'un échantillon d'entreprises françaises de taille importante publiant leurs états financiers en IFRS et de les 
comparer à ceux obtenus à partir de données French GAAPs divulguées par le même échantillon ou un groupe d'observations similaires. Deuxièmement, certains agrégats comptables établis à l'occasion du passage aux IFRS, comme les capitaux propres et le résultat net de la période, peuvent ne pas présenter une pertinence d'analyse suffisante en raison du caractère exceptionnel de certaines transactions qu'ils incluent pour l'année d'observation 2005. Sur un troisième point, notre argumentaire repose sur le fait que les principaux modèles de risque de défaut utilisés dans notre étude sont représentatifs des méthodologies d'évaluation retenues en France par les praticiens. Enfin la première application des normes comptables internationales est hétérogène au sein des groupes. En effet, conformément aux dispositions de la norme IFRS 1 ( $§ 13)$ certains groupes ont pu traiter de manière dérogatoire des éléments significatifs du bilan comme les instruments financiers (application prospective de la norme IAS 39) sans retraiter les comptes comparatifs 2004.

Les recherches futures pourraient s'intéresser à l'examen de davantage de modèles de risque de défaut (ex. modèle de la Banque de France) en vue de comparer nos résultats à ceux obtenus dans d'autres cadres d'analyse. D'autres recherches pourraient analyser le choix des options comptables appliqués lors de la première adoption du référentiel IFRS par rapport à la mesure du risque de défaut. 


\section{Bibliographie et références}

ALTMAN E. (1968), «Financial Ratios, Discriminant Analysis and the Prediction of Corporate Bankruptcy », Journal of Finance, 23, pp. 589-609.

ALTMAN E. et MCGOUGH T.P. (1974), «Evaluation of a Company as a Going Concern », Journal of Accountancy, décembre, pp. 50-57.

BARTH M., BEAVER W. et LANDSMAN W. (1998), «Relative Valuation Roles of Equity Book Value and Net Income as a Function of Financial Health », Journal of Accounting and Economics, 25, pp. 1-34.

BARDOS M. (1998), « Detecting the Risk of a Company Failure at the Banque de France », Journal of Banking \& Finance, 22, pp. 1405-1419.

BEAVER W. (1966), «Financial Ratios as Predictors of Bankruptcy », Journal of Accounting Research, 6, pp. 71-102.

BEAVER W. (1968), « Market Prices, Financial Ratios, and the Prediction of Failure », Journal of Accounting Research, 8, pp. 179-192.

BEGLEY J., MING J. et WATTS S. (1996), « Bankruptcy Classification Errors in the 1980's: An Empirical Analysis of Altman's and Ohlson's Models », Review of Accounting Studies, 1, pp. 267-284.

BELKAOUI A. (1984), «Théorie Comptable », Presses de l'Université du Québec, CP 250, Sillery, Québec G1T2R1.

BILLINGS B. (1999), «Revisiting the Relation between the Default Risk of Debt and the Earnings Response Coefficient », The Accounting Review, 74, 4, pp. 509-522.

BLACK, F. et SCHOLES M. (1973), «The Pricing of Options and Corporate Liabilities », Journal of Political Economy, 7, pp. 637-654.

BURGSTAHLER D., JIAMBALVO J. et NOREEN E. (1989), «Changes in the Probability of Bankruptcy and Equity Value », Journal of Accounting and Economics, 11, pp. 207-224.

CHEUNG J. (1991), «A Review of Option-Pricing Theory in Accounting Research », Journal of Accounting Literature, 10, pp. 51-84.

COLASSE B. (2001), «Comptabilité générale (PCG 1999 et IAS) », Economica, 7 ème édition, $471 \mathrm{p}$.

COLETTE C. et RICHARD J. (2000), «Comptabilité générale, les systèmes français et anglo-saxons », 660 p., Dunod

CORE J. et SCHRAND C. (1999), « The Effect of Accounting-Based Debt Covenants on Equity Valuation », Journal of Accounting and Economics, 27, 1, pp. 1-34. 
DAMBOLENA I. et KHOURY S. (1980), « Ratio Stability and Corporate Failure », Journal of Finance, 35, 4, pp. 1017-1026.

DE ANGELO H., DE ANGELO L. ET SKINNER D.J. (1994), «Accounting Choice in Troubled Companies », Journal of Accounting and Economics, Tome 17, pp. 113-143.

DHALIWAL D. et REYNOLDS S. (1994), « The Effect of the Default Risk of Debt on the Earnings Response Coefficient », The Accounting Review, 69, 2, pp. 412-419.

DICHEV I. (1998), « Is the Risk of Bankruptcy a Systematic Risk ? », Journal of Finance, 53, 3, pp. 1131-1147.

FAMA, E. et FRENCH K. (1997), «Industry Costs of Equity », Journal of Financial Economics, 43, 2, pp. 153-193.

FOSTER G. (1986), «Financial Statement Analysis », Prentice Hall, chapitre 15, pp. 553572 .

FRANCIS J. (1990), « Corporate Compliance with Debt Covenants », Journal of Accounting Research, 28, pp. 326-347.

GESKE, R. (1977), « The Valuation of Corporate Liabilities as Compound Options », Journal of Financial and Quantitative Analysis, 12, 4, pp. 541-552.

GRIFFIN, J. et LEMMON M. (2002), « Book-to-Market Equity, Distress Risk, and Stock Returns », Journal of Finance, 57, 5, pp. 2317-2336.

HAN, B., JENNINGS R. et NOEL J. (1992), « Communication of Nonearnings Information at the Financial Statements Release Date », Journal of Accounting and Economics, 15, 1, pp. 63-86.

HILLEGEIST S.A., KEATING E.K. et LUNDSTEDT K.G. (2004), "Assessing the Probability of Bankruptcy », Review of Accounting Studies, 9, pp. 5-34.

KOH H.C. et KILLOUGH L.N. (1990), « The use of Multiple Discriminant Analysis in the Assessment of the Going - Concern Status », Journal of Business, Finance Accounting, printemps, pp 179-192.

KOH H.C. (1991), « Model Predictions and Auditor Assessments of Going Concern Status », Accounting and Business Research, 21 (84), pp. 331-338.

MENSAH Y.H. (1983), «Price Level Adjustments: Some empirical Evidence», The Accounting Review, avril, pp. 228-248.

MERTON R. (1974), « On the Pricing of Corporate Debt: The Risk Structure of Interest Rates », Journal of Finance, 29, pp. 449-470.

OHLSON, J. (1980), «Financial Ratios and the Probabilistic Prediction of Bankruptcy » Journal of Accounting Research, 19, pp. 109-131. 
OPLER T. et TITMAN S. (1994), « Financial Distress and Corporate Performance », Journal of Finance, 49, 3, pp. 1015-1040.

PALEPU K. (1986), « Predicting Takeover Targets: A Methodological and Empirical Analysis », Journal of Accounting and Economics, 8, pp. 3-35.

PLATT H. et M. PLATT M. (1991), « A Note on the Use of Industry-Relative Ratios in Bankruptcy Prediction », Journal of Banking and Finance, 15, pp. 1183-1194.

ROGERS W. (1993), « Regression Standard Errors in Clustered Samples », Stata Technical Bulletin, 13, pp. 88-94.

RAFFOURNIER B. (2005), "Les normes comptables internationales (IFRS / IAS)», Economica, $2^{\text {ème }}$ édition, $523 \mathrm{p}$.

SABOLY M. (2001), "Information comptable et défaillance des enterprises : le cas français », Comptabilité-Contrôle-Audit, novembre, 7(2), pp.67-86.

SCHWARTZ K.B. (1982), «Accounting Changes by Corporations Facing Possible Insolvency », Journal of Accounting, Auditing and Finance, Automne, pp. 32-43.

SLOAN R. (1996), « Do Stock Prices Fully Reflect Information in Accruals and Cash Flows About Future Earnings ? », The Accounting Review, 71, 3, pp. 289-315.

TELLER R. (1995), «Essai sur la discontinuité comptable », dans « Mélange en l'honneur du Professeur Claude Pérochon », Foucher, pp. 495-506.

VASSALOU M. et XING Y. (2004), «Default Risk in Equity Returns”, Journal of Finance, Vol. 44(2), Avril 2004: 831-68.

VILANOVA (1997), «La décision de prêt bancaire comme signal imparfait sur l'emprunteur », Revue d'Economie Financière, ${ }^{\circ}$ 42, juillet, pp. 217-244.

WHITE G.I., SONDHI A.C. et FRIED D. (2002), « The Analysis and Use of Financial Statements $», 3^{\text {ème }}$ édition, Wiley International ed., $767 p$.

ZMIJEWSKI M.E. (1984), «Methodological Issues Related to the Estimation of Financial Distress Prediction Models », Journal of Accounting Research, supplément, 22, pp. 59-81. 
Tableau 1. Principales variables des modèles comptables prédictifs du risque de défaut

\begin{tabular}{|c|c|c|c|}
\hline \multirow[b]{2}{*}{ Indicateurs } & \multicolumn{3}{|c|}{ Modèles } \\
\hline & Altman (1968) & Ohlson (1980) & Zmijewski (1984) \\
\hline 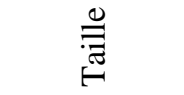 & - & $\begin{array}{l}\text { Log (total actifs / } \\
\text { indice du niveau des prix) }\end{array}$ & - \\
\hline \multirow{2}{*}{ : } & $\begin{array}{l}\text { BFR / } \\
\text { total des actifs }\end{array}$ & $\begin{array}{l}\text { BFR / } \\
\text { total des actifs }\end{array}$ & - \\
\hline & $\begin{array}{c}\text { Chiffre d'affaires / } \\
\text { total des actifs } \\
\text { (capacité de générer les actifs) }\end{array}$ & $\begin{array}{l}\text { Passifs courants / } \\
\text { Actifs courants }\end{array}$ & $\begin{array}{l}\text { Actifs courants / } \\
\text { Passifs courants }\end{array}$ \\
\hline \multirow{3}{*}{ 啻 } & $\begin{array}{l}\text { Résultats mis en réserves / } \\
\text { total des actifs } \\
\text { (profitabilité cumulative) }\end{array}$ & $\begin{array}{l}\text { Résultat net / } \\
\text { total des actifs } \\
\quad(\text { ROA })\end{array}$ & $\begin{array}{l}\text { Résultat net / } \\
\text { total des actifs } \\
\quad(\text { ROA })\end{array}$ \\
\hline & $\begin{array}{c}\text { EBIT (ou EBE) / } \\
\text { total des actifs } \\
\text { (profitabilité actuelle) }\end{array}$ & $\begin{array}{c}1 \text { si le résultat net a été négative au } \\
\text { cours des deux dernières années; } \\
0 \text { autrement }\end{array}$ & - \\
\hline & - & $\begin{array}{l}\text { Variation de résultat net }(\mathrm{RN}) \text { mesuré } \\
\text { comme } \\
=\frac{R N_{t}-R N_{t-1}}{\left|R N_{t}\right|+\left|R N_{t-1}\right|}\end{array}$ & - \\
\hline \multirow{3}{*}{ 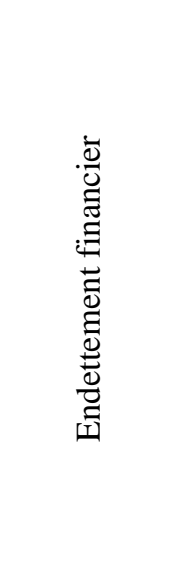 } & $\begin{array}{l}\text { Market value equity / book value } \\
\text { of debt (current and long term } \\
\text { debts) } \\
\text { (assets value decline capacity) }\end{array}$ & $\begin{array}{l}\text { Total des passifs / } \\
\text { total des actifs }\end{array}$ & $\begin{array}{l}\text { Total des dettes / } \\
\text { total des actifs }\end{array}$ \\
\hline & - & $\begin{array}{c}1 \text { si total des passifs (hors capitaux } \\
\text { propres) }>\text { total des actifs; } \\
0 \text { autrement } \\
\text { (correcteur de discontinuité de } \\
\text { l'endettement financier) }\end{array}$ & - \\
\hline & - & $\begin{array}{l}\text { Flux de trésorerie opérationnels / total } \\
\text { des passifs (hors capitaux propres) }\end{array}$ & - \\
\hline
\end{tabular}

Le tableau 1 présente les variables comptables d'analyse sélectionnées par Altman (1968), Ohlson (1980) et Zmijewski (1984) dans leurs modèles comptables respectifs de prédiction du risque de défaut. Ces variables sont classées par groupe d'indicateurs suivant la classification élaborée par White et al. (2002). Conformément à l'analyse de ces auteurs, le Besoin en Fonds de Roulement est calculé comme la différence entre les actifs courants et les passifs courants. 
Tableau 2. Ventilation sectorielle (niveau 2 de l'ICB) de l'échantillon d'analyse

\begin{tabular}{llrr}
\hline \hline ICB Sector (Level 2) & N & \% \\
\hline 0500 & Oil \& Gas & 2 & 2 \\
1300 & Chemicals & 2 & 2 \\
1700 & Basic Resources & 3 & 3 \\
2300 & Construction \& Materials & 8 & 9 \\
2700 & Industrial Goods \& Services & 22 & 24 \\
3300 & Automobiles \& Parts & 5 & 6 \\
3500 & Food \& Beverage & 4 & 4 \\
3700 & Personal \& Household Goods & 9 & 10 \\
4500 & Health Care & 2 & 2 \\
5300 & Retail & 4 & 4 \\
5500 & Media & 11 & 12 \\
5700 & Travel \& Leisure & 4 & 4 \\
6500 & Telecommunications & 1 & 1 \\
7500 & Utilities & 5 & 6 \\
9500 & Technology & 8 & 9 \\
& Total & $\mathbf{9 0}$ & $\mathbf{1 0 0}$ \\
\hline
\end{tabular}

Le tableau 2 présente une ventilation de l'échantillon d'analyse par secteur industriel basée sur la classification INDC6 (niveau le plus détaillé de DATASTREAM®). Cette analyse industrielle est basée sur la nouvelle classification FTSE/DJ (similaire au schéma de classification de $4^{\text {ème }}$ degré US SIC 4) et comprend 83 sous-sections sectorielles. La troisième colonne du tableau indique le nombre d'entreprises échantillonnées par secteur et la quatrième colonne présente le pourcentage représenté par le secteur dans l'échantillon final. 
Tableau 3. Statistiques descriptives des variables comptables en référentiel français et international IAS / IFRS

\begin{tabular}{|c|c|c|c|c|c|c|}
\hline Variable (en M€) & $N$ & Mean & Median & StdDev & Skewness & Kurtosis \\
\hline \multicolumn{7}{|l|}{ Chiffre d'affaires } \\
\hline - selon PCG & 73 & 14255,77 & 7994,00 & 19297,18 & 3,21 & 13,97 \\
\hline - selon référentiel IAS / IFRS & 73 & 13757,64 & 7787,00 & 19082,89 & 3,31 & 14,65 \\
\hline Variation par observation (en \%) & 73 & $-4,17$ & $-0,42$ & 9,73 & $-3,92$ & 19,89 \\
\hline \multicolumn{7}{|l|}{ Total actifs } \\
\hline - selon PCG & 73 & 19129,15 & 9721,00 & 25901,41 & 2,68 & 8,90 \\
\hline - selon référentiel IAS / IFRS & 73 & 19641,63 & 8961,70 & 27003,58 & 2,62 & 8,23 \\
\hline Variation par observation (en \%) & 73 & 2,12 & 1,36 & 11,45 & $-2,94$ & 13,98 \\
\hline \multicolumn{7}{|c|}{ Total des passifs (hors capitaux propres) } \\
\hline - selon PCG & 70 & 11753,69 & 6479,50 & 14567,08 & 2,16 & 5,50 \\
\hline - selon référentiel IAS / IFRS & 70 & 12597,64 & 5968,00 & 15785,18 & 2,17 & 5,37 \\
\hline Variation par observation (en \%) & 70 & 8,09 & 4,95 & 17,65 & 0,06 & 3,58 \\
\hline \multicolumn{7}{|l|}{ Total des actifs courants } \\
\hline - selon PCG & 70 & 7039,53 & 4275,65 & 7797,93 & 1,96 & 4,30 \\
\hline - selon référentiel IAS / IFRS & 70 & 6582,64 & 4065,55 & 7595,33 & 2,15 & 5,09 \\
\hline Variation par observation (en \%) & 70 & $-5,08$ & $-3,44$ & 14,05 & $-0,75$ & 6,52 \\
\hline \multicolumn{7}{|l|}{ Total des passifs courants } \\
\hline - selon PCG & 23 & 8124,59 & 5933,00 & 8050,65 & 1,56 & 2,32 \\
\hline - selon référentiel IAS / IFRS & 23 & 9148,79 & 6687,00 & 8366,60 & 1,19 & 1,06 \\
\hline Variation par observation (en \%) & 23 & 27,90 & 1,90 & 110,85 & 4,70 & 22,34 \\
\hline \multicolumn{7}{|c|}{ Besoin en Fonds de Roulement (BFR) } \\
\hline - selon PCG & 73 & 66,21 & 157,30 & 3008,75 & 1,36 & 11,67 \\
\hline - selon référentiel IAS / IFRS & 73 & $-198,34$ & 5,60 & 3466,85 & $-0,67$ & 10,85 \\
\hline Variation par observation (en \%) & 73 & $-34,81$ & $-8,33$ & 155,62 & $-3,17$ & 13,87 \\
\hline \multicolumn{7}{|l|}{ Résultat net } \\
\hline - selon PCG & 70 & 561,22 & 215,10 & 1434,05 & 3,72 & 23,88 \\
\hline - selon référentiel IAS / IFRS & 70 & 765,29 & $269,50 *$ & 1518,85 & 4,71 & 28,60 \\
\hline Variation par observation (en \%) & 70 & 72,76 & 13,82 & 301,17 & 6,29 & 43,72 \\
\hline
\end{tabular}




\begin{tabular}{|c|c|c|c|c|c|c|}
\hline Variable (en M€) & $N$ & Mean & Median & StdDev & Skewness & Kurtosis \\
\hline \multicolumn{7}{|l|}{ EBIT (ои EBE) } \\
\hline - selon PCG & 73 & 1314,52 & 530,00 & 2347,49 & 4,57 & 24,69 \\
\hline - selon référentiel IAS / IFRS & 73 & 1308,79 & 519,00 & 2374,01 & 4,62 & 25,96 \\
\hline Variation par observation (en \%) & 73 & $-28,59$ & $-5,21$ & 124,45 & $-6,08$ & 38,42 \\
\hline \multicolumn{7}{|l|}{ Total des dettes } \\
\hline - selon PCG & 70 & 10209,18 & 5662,40 & 12685,11 & 2,36 & 7,34 \\
\hline - selon référentiel IAS / IFRS & 70 & 11212,42 & 5494,50 & 14106,69 & 2,34 & 7,07 \\
\hline Variation par observation (en \%) & 70 & 11,13 & 5,15 & 21,94 & 0,50 & 2,69 \\
\hline \multicolumn{7}{|l|}{ Total des dettes financiers à $C T$} \\
\hline - selon PCG & 68 & 794,24 & 7,50 & 1855,63 & 3,82 & 17,34 \\
\hline - selon référentiel IAS / IFRS & 68 & $1682,59 * * *$ & $383,30 * * *$ & 3459,02 & 4,38 & 23,07 \\
\hline Variation par observation (en \%) & 68 & 32,84 & 5,40 & 117,81 & 3,74 & 16,23 \\
\hline \multicolumn{7}{|l|}{ Total des dettes non financiers } \\
\hline - selon PCG & 72 & 5421,98 & 2836,40 & 6722,43 & 2,08 & 5,24 \\
\hline - selon référentiel IAS / IFRS & 72 & 5373,10 & 2643,10 & 6740,49 & 1,95 & 3,81 \\
\hline Variation par observation (en \%) & 72 & 0,82 & 0,00 & 20,19 & $-0,12$ & 4,27 \\
\hline \multicolumn{7}{|l|}{ Total des créances } \\
\hline - selon PCG & 73 & 3931,10 & 2128,00 & 5035,12 & 2,35 & 6,00 \\
\hline - selon référentiel IAS / IFRS & 73 & 3497,19 & 1988,00 & 4551,45 & 2,70 & 8,29 \\
\hline Variation par observation (en \%) & 73 & $-5,83$ & $-6,23$ & 17,62 & 0,95 & 3,27 \\
\hline
\end{tabular}

Le tableau 3 présente les statistiques univariées pour les agrégats comptables utiles à la construction des variables indépendantes des modèles prédictifs de défaut. L'échantillon comprend toutes les entreprises industrielles composant l'indice SBF 120 dont les données nécessaires au processus de modélisation sont disponibles à partir de leurs rapports annuels respectifs au 31 décembre 2004 . Les observations extrêmes comprises dans la tranche des $1 \%$ supérieurs ou inférieurs de la distribution sont éliminées de l'échantillon afin d'éviter des problèmes potentiels de biais par "outliers". Les astérisques indiquent que les moyennes (médianes) des variables en PCG et en IAS / IFRS sont significativement l'une de l'autre lorsqu'on utilise un test- $t$ bilatéral (test de Mann-Whitney-Wilcoxon).

Les notations suivantes sont utilisées: ${ }^{*} \mathrm{p}<.1 ; * * \mathrm{p}<.05 ; * * \mathrm{p}<.01$. 
Tableau 4. Statistiques descriptives des variables de modèle comptable

\begin{tabular}{|c|c|c|c|c|c|c|}
\hline Variable & $\bar{N} N$ & Mean & Median & StdDev & Skewness & Kurtosis \\
\hline \multicolumn{7}{|l|}{ (1) Taille } \\
\hline \multicolumn{7}{|l|}{$\log ($ total actifs $)$} \\
\hline - selon PCG & 73 & 3,95 & 3,99 & 0,57 & 0,01 & $-0,88$ \\
\hline - selon référentiel IAS / IFRS & 73 & 3,95 & 3,95 & 0,57 & 0,05 & $-0,85$ \\
\hline Variation par observation (en \%) & 73 & 0,15 & 0,16 & 1,48 & $-4,05$ & 20,46 \\
\hline \multicolumn{7}{|l|}{ (2) Liquidité } \\
\hline \multicolumn{7}{|l|}{ BFR / Total actifs } \\
\hline - selon PCG & 73 & 0,04 & 0,04 & 0,13 & 0,52 & 0,26 \\
\hline - selon référentiel IAS / IFRS & 73 & 0,03 & 0,01 & 0,15 & 0,54 & 1,77 \\
\hline Variation par observation (en \%) & 73 & $-33,62$ & $-10,54$ & 151,62 & $-3,12$ & 13,66 \\
\hline \multicolumn{7}{|l|}{ CA / Total actifs } \\
\hline - selon PCG & 73 & 0,85 & 0,77 & 0,38 & 0,80 & 0,54 \\
\hline - selon référentiel IAS / IFRS & 73 & 0,81 & 0,76 & 0,40 & 1,24 & 2,54 \\
\hline Variation par observation (en \%) & 73 & $-4,01$ & $-3,56$ & 22,97 & 3,71 & 20,57 \\
\hline \multicolumn{7}{|l|}{ Passifs courants / actifs courants } \\
\hline - selon PCG & 23 & 0,83 & 0,78 & 0,38 & 1,09 & 2,52 \\
\hline - selon référentiel IAS / IFRS & 23 & 0,91 & 0,81 & 0,39 & 1,58 & 4,08 \\
\hline Variation par observation (en \%) & 23 & 30,83 & 8,16 & 124,21 & 4,69 & 22,32 \\
\hline \multicolumn{7}{|l|}{ (3) Profitabilité } \\
\hline \multicolumn{7}{|l|}{ Résultat net / total actifs (ROA) } \\
\hline - selon PCG & 70 & 0,04 & 0,03 & 0,05 & 0,42 & 2,08 \\
\hline - selon référentiel IAS / IFRS & 70 & $0,05 *$ & 0,04 & 0,05 & 0,65 & 2,84 \\
\hline Variation par observation (en \%) & 70 & 104,53 & 10,36 & 565,90 & 7,70 & 61,86 \\
\hline \multicolumn{7}{|l|}{ EBIT (ou EBE) / total actifs } \\
\hline - selon PCG & 73 & 0,09 & 0,08 & 0,06 & 1,29 & 2,45 \\
\hline - selon référentiel IAS / IFRS & 73 & 0,08 & 0,07 & 0,06 & 1,22 & 2,32 \\
\hline Variation par observation (en \%) & 73 & $-26,14$ & $-8,62$ & 114,88 & $-5,73$ & 35,88 \\
\hline
\end{tabular}




\begin{tabular}{|c|c|c|c|c|c|c|}
\hline Variable & $N$ & Mean & Median & StdDev & Skewness & Kurtosis \\
\hline \multicolumn{7}{|l|}{ Variation de résultat net (Ohlson) } \\
\hline $\begin{array}{l}\text { - selon PCG } \\
\text { - selon référentiel IAS / IFRS }\end{array}$ & $\begin{array}{l}68 \\
68\end{array}$ & $\begin{array}{r}0,14 \\
0,24 *\end{array}$ & $\begin{array}{r}0,12 \\
0,14 *\end{array}$ & $\begin{array}{l}0,46 \\
0,44\end{array}$ & $\begin{array}{l}-0,08 \\
-0,11\end{array}$ & $\begin{array}{l}1,10 \\
0,89\end{array}$ \\
\hline Variation par observation (en \%) & 68 & 23,36 & 0,00 & 294,27 & 0,16 & 10,66 \\
\hline \multicolumn{7}{|l|}{ (4) Endettement financier } \\
\hline \multicolumn{7}{|l|}{ Capitalisation boursière / total dettes } \\
\hline - selon PCG & 63 & 2,03 & 1,18 & 2,56 & 3,09 & 11,16 \\
\hline - selon référentiel IAS / IFRS & 63 & 1,84 & 1,00 & 2,31 & 2,89 & 9,75 \\
\hline Variation par observation (en \%) & 63 & $-7,43$ & $-5,28$ & 23,05 & 4,10 & 26,37 \\
\hline \multicolumn{7}{|l|}{ Total passifs / total actifs } \\
\hline - selon PCG & 73 & 0,67 & 0,69 & 0,16 & $-0,59$ & 0,08 \\
\hline - selon référentiel IAS / IFRS & 73 & 0,68 & 0,70 & 0,16 & $-0,87$ & 0,62 \\
\hline Variation par observation (en \%) & 73 & 1,12 & 1,19 & 10,21 & $-3,80$ & 25,70 \\
\hline \multicolumn{7}{|l|}{ Total dettes / total actifs } \\
\hline - selon PCG & 70 & 0,56 & 0,57 & 0,16 & $-0,15$ & $-0,81$ \\
\hline - selon référentiel IAS / IFRS & 70 & $0,61 *$ & $0,62 *$ & 0,17 & 0,28 & 1,15 \\
\hline Variation par observation (en \%) & 70 & 9,40 & 3,67 & 21,90 & 2,54 & 10,26 \\
\hline \multicolumn{7}{|c|}{ Total dettes financières court terme / total actifs } \\
\hline - selon PCG & 68 & 0,03 & 0,01 & 0,05 & 1,97 & 3,71 \\
\hline - selon référentiel IAS / IFRS & 68 & $0,07 * * *$ & $0,06 * * *$ & 0,07 & 1,86 & 4,66 \\
\hline Variation par observation (en \%) & 68 & 30,86 & 0,78 & 109,93 & 3,34 & 13,43 \\
\hline
\end{tabular}


Panel B. Coefficients de corrélation de Pearson/Spearman (rangs)

Echantillon PCG

\begin{tabular}{|c|c|c|c|c|c|c|c|c|c|c|c|}
\hline & TAILLE & LIQ1 & LIQ2 & LIQ3 & PROF1 & PROF2 & PROF3 & GEAR1 & GEAR2 & GEAR3 & GEAR4 \\
\hline TAILLE & 1.000 & -0.346 & -0.297 & 0.309 & -0.174 & -0.416 & 0.120 & -0.505 & 0.467 & 0.183 & 0.296 \\
\hline LIQ1 & -0.264 & 1.000 & 0.193 & -0.626 & 0.387 & 0.395 & 0.052 & 0.315 & -0.465 & -0.450 & -0.071 \\
\hline LIQ2 & -0.280 & 0.103 & 1.000 & -0.348 & 0.089 & 0.132 & -0.093 & 0.002 & -0.060 & 0.075 & -0.102 \\
\hline LIQ3 & 0.400 & -0.538 & -0.322 & 1.000 & -0.432 & -0.300 & 0.100 & -0.444 & 0.550 & 0.708 & 0.214 \\
\hline PROF1 & 0.206 & 0.395 & -0.110 & -0.282 & 1.000 & 0.792 & 0.038 & 0.569 & -0.459 & -0.378 & -0.178 \\
\hline PROF2 & -0.359 & 0.146 & 0.145 & -0.180 & 0.054 & 1.000 & -0.094 & 0.769 & -0.606 & -0.419 & -0.095 \\
\hline PROF3 & 0.066 & -0.022 & 0.015 & 0.104 & -0.110 & 0.076 & 1.000 & -0.174 & 0.217 & 0.185 & -0.092 \\
\hline GEAR1 & -0.361 & 0.118 & -0.057 & -0.404 & 0.028 & 0.342 & -0.111 & 1.000 & -0.827 & -0.629 & -0.023 \\
\hline GEAR2 & 0.448 & -0.309 & -0.010 & 0.529 & 0.022 & -0.307 & 0.290 & -0.731 & 1.000 & 0.788 & -0.001 \\
\hline GEAR3 & 0.187 & -0.402 & 0.074 & 0.737 & -0.031 & -0.203 & 0.229 & -0.568 & 0.800 & 1.000 & -0.049 \\
\hline GEAR4 & 0.246 & 0.012 & -0.001 & 0.225 & 0.059 & -0.115 & -0.033 & -0.146 & 0.082 & 0.063 & 1.000 \\
\hline
\end{tabular}

\section{Echantillon IAS}

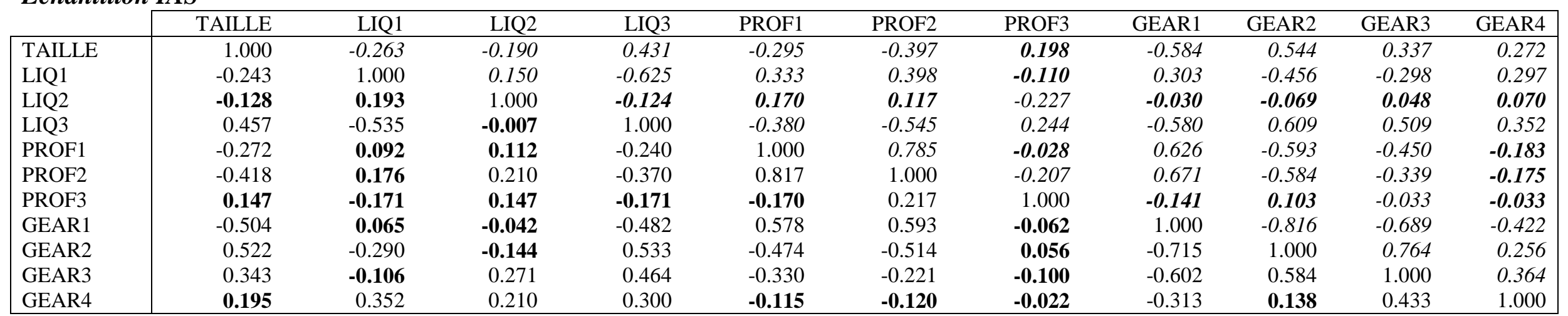

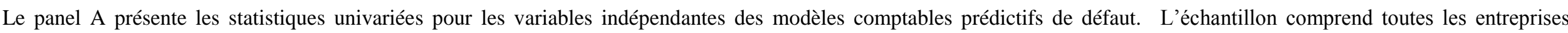

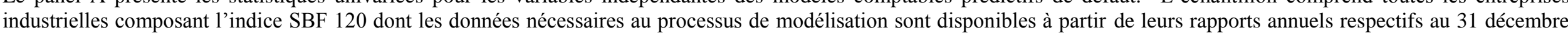

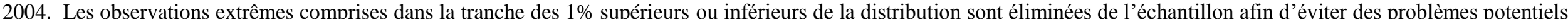

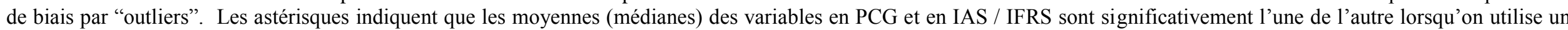

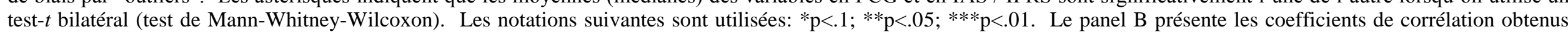

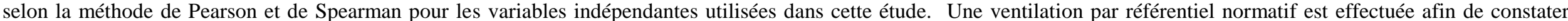

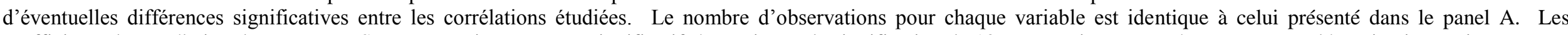

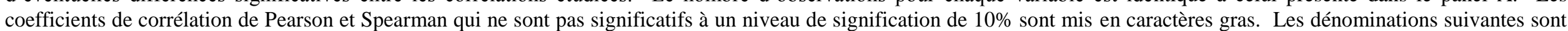

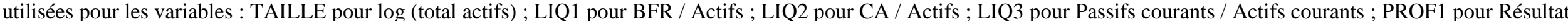

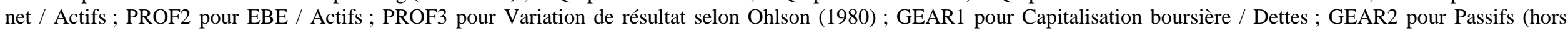
capitaux propres) / Actifs ; GEAR3 pour Dettes / Actifs ; GEAR4 pour Dettes financières à court terme / Actifs.

Proposition - Numéro spécial CCA 2007 
Tableau 5. Impacts de la transition aux IFRS sur les modèles optionnels de risque de défaut

Panel A : Sensibilité de la valeur des capitaux propres de l'entreprise selon le modèle de Merton

\begin{tabular}{|c|c|c|c|c|c|c|c|c|}
\hline \multirow{2}{*}{$\sigma_{\mathrm{A}}$} & \multicolumn{2}{|c|}{$5 \%$} & \multicolumn{2}{|c|}{$10 \%$} & \multicolumn{2}{|c|}{$15 \%$} & \multicolumn{2}{|c|}{$20 \%$} \\
\hline & $\begin{array}{c}\text { Moyenne } \\
-0,899471801\end{array}$ & $\begin{array}{c}\text { Médiane } \\
-0,975309912\end{array}$ & $\begin{array}{c}\text { Moyenne } \\
-0,880674484\end{array}$ & $\begin{array}{c}\text { Médiane } \\
-0,975309912\end{array}$ & $\begin{array}{c}\text { Moyenne } \\
-0,838118698\end{array}$ & $\begin{array}{c}\text { Médiane } \\
-0,975235937\end{array}$ & $\begin{array}{c}\text { Moyenne } \\
-0,769868593\end{array}$ & $\begin{array}{c}\text { Médiane } \\
-0,968817686\end{array}$ \\
\hline
\end{tabular}

Panel B : Sensibilité de la probabilité de défaut de l'entreprise selon le modèle de Merton

\begin{tabular}{|c|c|c|c|c|c|c|c|c|}
\hline \multirow{3}{*}{$\sigma_{\mathrm{A}}$} & \multicolumn{2}{|c|}{$5 \%$} & \multicolumn{2}{|c|}{$10 \%$} & \multicolumn{2}{|c|}{$15 \%$} & \multicolumn{2}{|c|}{$20 \%$} \\
\hline & Moyenne & Médiane & Moyenne & Médiane & Moyenne & Médiane & Moyenne & Médiane \\
\hline & $-6,3233 \mathrm{E}-06$ & $-2,23688 \mathrm{E}-42$ & $-7,29257 \mathrm{E}-06$ & $-2,60087 \mathrm{E}-13$ & $-1,41508 \mathrm{E}-05$ & $-3,49703 \mathrm{E}-08$ & $-2,62481 \mathrm{E}-05$ & $5 E-06$ \\
\hline $5 \%$ & $-2,03342 \mathrm{E}-06$ & $-2,07319 \mathrm{E}-48$ & $-4,17809 \mathrm{E}-06$ & $-8,41313 \mathrm{E}-15$ & $-8,54263 \mathrm{E}-06$ & -7,92479E-09 & $-1,76555 \mathrm{E}-05$ & 3E-07 \\
\hline $10 \%$ & $-2,7331 \mathrm{E}-07$ & $-7,0808 \mathrm{E}-55$ & $-1,40256 \mathrm{E}-08$ & $-8,91019 \mathrm{E}-62$ & $-4,9898 \mathrm{E}-06$ & -1,69494E-09 & $-1,15252 \mathrm{E}-05$ & 74E-07 \\
\hline $15 \%$ & $-1,40256 \mathrm{E}-08$ & $-8,91019 \mathrm{E}-62$ & $-8,99232 \mathrm{E}-07$ & $-3,91122 \mathrm{E}-18$ & $-2,80261 \mathrm{E}-06$ & $-2,9751 \mathrm{E}-10$ & -7,30068E-06 & $-1,04052 \mathrm{E}-07$ \\
\hline $20 \%$ & $-2,67382 \mathrm{E}-10$ & $-4,13028 \mathrm{E}-69$ & $-3,10985 \mathrm{E}-07$ & $-5,79836 \mathrm{E}-20$ & $-1,49798 \mathrm{E}-06$ & $-4,3611 \mathrm{E}-11$ & $-4,48635 \mathrm{E}-06$ & $-3,84792 \mathrm{E}-08$ \\
\hline
\end{tabular}

Panel C : Variation de la probabilité de défaut de l'entreprise liée au passage aux normes IFRS selon le modèle de Merton

\begin{tabular}{|c|c|c|c|c|c|c|c|c|}
\hline \multirow{3}{*}{$\sigma_{\mathrm{A}}$} & \multicolumn{2}{|c|}{$5 \%$} & \multicolumn{2}{|c|}{$10 \%$} & \multicolumn{2}{|c|}{$15 \%$} & \multicolumn{2}{|c|}{$20 \%$} \\
\hline & Moyenne & Médiane & Moyenne & Médiane & Moyenne & Médiane & Moyenne & Médiane \\
\hline & $-0,414 \%$ & $-1,56739 \mathrm{E}-72$ & $-0,763 \%$ & $-3,97997 \mathrm{E}-17$ & $-0,456 \%$ & $-5,48354 \mathrm{E}-07$ & $-0,486 \%$ & $-0,000192023$ \\
\hline $5 \%$ & $-0,040 \%$ & $-9,05331 \mathrm{E}-81$ & $-0,442 \%$ & $-3,53533 \mathrm{E}-19$ & $-0,409 \%$ & $-6,81299 \mathrm{E}-08$ & $-0,383 \%$ & $-5,50139 \mathrm{E}-05$ \\
\hline $10 \%$ & $-0,001 \%$ & -1,92372E-89 & $-0,195 \%$ & $-2,44572 \mathrm{E}-21$ & $-0,316 \%$ & -7,57459E-09 & $-0,307 \%$ & $-1,66991 \mathrm{E}-05$ \\
\hline $15 \%$ & $0,000 \%$ & $-1,50378 \mathrm{E}-98$ & $-0,067 \%$ & $-1,31768 \mathrm{E}-23$ & $-0,212 \%$ & $-7,53574 \mathrm{E}-10$ & $-0,241 \%$ & $-5,98044 \mathrm{E}-06$ \\
\hline $20 \%$ & $0,000 \%$ & $-4,3244 \mathrm{E}-108$ & $-0,018 \%$ & $-5,52891 \mathrm{E}-26$ & $-0,124 \%$ & $-6,70869 \mathrm{E}-11$ & $-0,182 \%$ & $-2,01201 \mathrm{E}-06$ \\
\hline Moyenne & $-0,09 \%$ & $0,00 \%$ & $-0,30 \%$ & $0,00 \%$ & $-0,30 \%$ & $0,00 \%$ & $-0,32 \%$ & $-0,01 \%$ \\
\hline
\end{tabular}

Le tableau 5 présente les résultats de l'analyse de l'impact de la transition aux normes IFRS sur l'estimation de la probabilité de défaut d'une entreprise par le modèle optionnel de Merton (1974) tel qu'adapté par Vassalou et Xing (2004). Les panels A et B du tableau 5 présentent respectivement les sensibilités de la valeur des capitaux propres estimés selon le modèle et de la probabilité de défaut à la variation de l'endettement de l'entreprise suivant différents scenarii de la volatilité, $\sigma_{\mathrm{A}}$, et de la croissance, $\mu_{\mathrm{A}}$, des actifs de l'entreprise. Le panel $\mathrm{C}$ présente la variation moyenne et médiane des probabilités de défaut des entreprises échantillonnées selon ces différents scenarii. 


\section{NOTES DE FIN}

${ }^{1}$ CNCC (2003) : « Norme n ${ }^{\circ}$ 6-702 - Alerte ».

${ }^{2}$ Loi du 24 juillet 1966 modifiée par la loi du $1^{\text {er }}$ mars 1984, art. 230-1 et 230-2.

${ }^{3}$ IASB (2005) : Cadre conceptuel ( $\$ 23$ )

${ }^{4} C f$. IAS 1 paragraphes 23 et 24 .

${ }^{5}$ Le cadre conceptuel ( $\left.\$ 89\right)$ définit un actif en fonction notamment de sa rentabilité : « un actif est comptabilisé au bilan lorsqu'il est probable que les avantages économiques futures iront à l'entité...».

${ }^{6}$ Les sociétés cotées ont en effet publiées un 31 décembre 2004 IFRS comparatif pour les comptes IFRS clos au 31 décembre 2005. En 2004, les sociétés avaient publiées des comptes PCG pour l'exercice clos au 31 décembre 2004.

${ }^{7}$ CNCC (1996) : Bulletin d'information $n^{\circ} 102$, juin

${ }^{8}$ SFAC 5 : « Recognition and Measurement in Financial Statements of Business Enterprises ».

${ }^{9}$ IASB (2006) : Framework ( $\left.\$ 23\right)$

${ }^{10}$ Les auteurs font référence au coût historique pour définir la comptabilité dynamique et à la valeur de marché pour les actifs pris chacun séparément. Suivant cette typologie, le PCG 1982 avant les transpositions IFRS est de type dynamique tandis que les normes IFRS seraient proches d'une comptabilité statique et actuarielle. La comptabilisation d'un actif sur la base d'une valeur d'utilité pour réaliser un test de dépréciation est de type actuariel alors que l'évaluation des titres de placement à la valeur de marché à la clôture est de type statique.

${ }^{11}$ Richard et Colette (2000, p.55) postulent que «dans les conditions concrètes actuelles des législations comptables, l'hypothèse de continuité d'exploitation n'a aucun impact sur les modalités de l'évaluation; celle-ci reste statique ou actuarielle comme dans l'hypothèse inverse de la non continuité ».

${ }^{12}$ Rappelons que l'indice SBF 120, lancé le 8 décembre 1993, est composé des 40 valeurs de l'indice CAC 40 auxquelles s'ajoutent 80 valeurs du premier marché parmi les plus liquides.

${ }^{13}$ En son paragraphe 6, la norme IFRS 1 dispose qu' " une entité est tenue de préparer un premier bilan d'ouverture en IFRS à la date de transition aux Normes. Celui-ci est le point de départ de sa comptabilité selon les Normes. [...] ». Cette disposition normative nous permet de pouvoir comparer le bilan de clôture 2004 établi selon les French GAAPs avec le bilan d'ouverture 2005 établi selon les normes IAS / IFRS.

${ }^{14}$ Cette classification est celle couramment retenue par les bases de données financières traditionnelles (e.g. Thomson Financial DataStream ${ }^{\circledR}$, Bloomberg $\left.®\right)$. Euronext base également son analyse sectorielle sur cette classification.

${ }^{15}$ Suivant les enseignements du modèle KMV et de Vassalou et Xing (2004) et Hillegeist et al. (2004), nous avons également réalisé les tests en égalisant le prix d'exercice de l'option aux passifs courants et à la moitié des passifs non courants. Les résultats se sont avérés globalement identiques à ceux présentés dans le tableau 5. 